\title{
Terceirização em cuidados continuados - uma abordagem de gestão de risco
}

\author{
Outsourcing in long-term care - a risk management approach
}

Cristina Machado Guimarães ${ }^{1}$

José Crespo de Carvalho ${ }^{1}$

${ }^{1}$ Instituto Universitário de Lisboa (ISCTE). Av. Forças Armadas s/n, Campo

Grande. 1649-026 Lisboa Portugal.

cristinamachadoguimaraes

@gmail.com
Abstract This article seeks to investigate outsourcing decisions in supply chain management of healthcare organizations, namely the motives and constraints behind the decision, the selection criteria for activities to be outsourced to third parties, the type of possible agreements, and the impact of this decision on the organization per se. A case study of the start-up phase of a Longterm Care unit with an innovative approach and high levels of customization was conducted to understand the outsourcing process in a start-up context (not in the standard context of organizational change) and a risk evaluation matrix was created for outsourcing activities in order to define and implement a performance monitoring process. This study seeks to understand how to evaluate and assess the risks of an outsourcing strategy and proposes a monitoring model using risk management tools. It was shown that the risk management approach can be a solution for monitoring outsourcing in the organizational startup phase. Conclusions concerning dissatisfaction with the results of outsourcing strategies adopted are also presented.

Key words Healthcare, Performance monitoring, Outsourcing, Risk management, Long-term care
Resumo Este artigo tem como objetivo investigar a decisão de externalização, integrada na gestão da cadeia de suprimentos no setor da saúde, nomeadamente os motivos e condicionantes da decisão, os critérios de escolha das atividades a entregar a terceiros, os tipos de acordos possiveis e o impacto que a decisão tem na organização e na pratica. Através do estudo de caso de uma unidade de saúde prestadora de cuidados continuados de forma inovadora, com grandes níveis de customização e em início de atividade, investigou-se todo o processo de terceirização em contexto do início do ciclo de vida de uma organização (não no contexto comum de mudança organizacional) e construiu-se uma matriz de avaliação de risco para atividades terceirizadas de modo a permitir a definição do processo de monitorização da performance a implementar. Este estudo visa compreender como se avaliam e balizam os riscos da adopção de uma estratégia de terceirização e sugere um modelo de monitorização, com recurso a ferramentas de gestão de risco. Concluiu-se que uma abordagem de Gestão de Risco pode ser uma solução para monitorização de terceirização em atividade que se iniciam. Retiram-se, ainda, conclusões acerca da insatisfação com os resultados da estratégia de externalização adotada.

Palavras-chave Saúde, Monitorização da performance, Terceirização, Gestão de risco, Cuidados continuados 


\section{Introdução}

A tendência crescente de terceirização no setor da saúde ${ }^{1-3}$ demonstra um alargamento do espetro do fenômeno ao longo das duas últimas décadas. Se inicialmente se resumia a encontrar uma entidade externa à organização que executasse a mesma tarefa, simultaneamente de forma mais rápida, melhor e a menor custo, a evidência aponta para uma evolução do conceito passando a serem reconhecidos como estratégicos os motivos que levam à terceirização como libertadora de recursos necessários a atividades de maior valor acrescentado ${ }^{4}$. Mais recentemente, a terceirização, para além de cumprir os anteriores propósitos, é olhada como fonte de inovação que origina quer novas formas de fazer o mesmo negócio, quer novos negócios ${ }^{5,6}$. Os fatores determinantes da decisão de externalizar não são consensuais num setor onde o epicentro é o usuário e/ou a família e onde os serviços que impliquem um contato direto podem ser vistos como críticos e de difícil externalização. Optar pela terceirização de atividades não deve ser uma panaceia quando os resultados se traduzem no bemestar dos utentes/usuários com elevadas expectativas na qualidade da prestação.

Neste artigo, ao investigar-se a terceirização em contexto de organizações de saúde, procurase aferir da satisfação com a decisão como resultado de uma correcta avaliação dos riscos e benefícios e de uma boa gestão de todo o processo, realçando o papel da monitorização das atividades externalizadas como garantia da performance. Dando ênfase às vicissitudes próprias da prática de terceirização em unidades de saúde, esta investigação, mais do que mapear as vantagens e riscos associados à decisão, analisa a importância da monitorização da performance das atividades externalizadas como forma de balizar os riscos inerentes. O desafio é, através do estudo do caso de uma unidade de saúde no início de atividade, olhar para a monitorização da performance das atividades externalizadas, não pela ótica das conformidades, da verificação de índices perante indicadores pré-definidos, mas pela ótica da construção de indicadores quando ainda não se encontram definidos ou suscitam acertos. A ideia subjacente é o exercício de "o que pode correr mal?", pertinente nos começos de atividade.

Apesar da parca investigação publicada sobre a extensão da terceirização em serviços de saúde, a literatura existente ${ }^{7}$ remete para a necessidade de investigação empírica sobre as medidas e os modelos de performance a adotar perante as especificações correntes nos contratos de terceirização, uma vez que não fornece respostas para várias perguntas, aqui tratadas como Questões de Investigação (QI). A literatura é também omissa quanto a práticas de terceirização em início de atividade, nomeadamente em serviços de saúde. Num setor que enfrenta grandes desafios, a realidade dos cuidados continuados não só merece ser estudada pelas alterações demográficas da sociedade como pela morbilidade a elas associada. Opções com vista apenas a resultados imediatos em custos podem tornar a equação de valor inadequada a um usuário que permaneça bastante mais tempo nestas unidades de saúde que em outras diferentes.

\section{Terceirização em organizações de saúde}

As organizações de saúde recorrem à terceirização por algumas razões similares às de outras noutros setores de actividade ${ }^{2}$ embora com consequências diferentes, nomeadamente em termos de fiabilidade e performance. Os motivos mais mencionados na literatura são: redução de custos, mitigação do risco, operação de mudanças rápidas sem comprometer recursos internos ${ }^{4,8}$. As expectativas de redução de custos podem, no entanto, ser defraudadas devido a uma má avaliação dos chamados "custos escondidos", os custos indiretos (adjudicação, custos de transição, de maus contratos e de monitorização) e os custos sociais (redução da "moral", baixa produtividade e alta rotação de colaboradores) $)^{9}$. Para além dos motivos financeiros, tecnológicos, estratégicos e políticos há a ter em conta a arquitetura do serviço (mais modular ou mais integral $)^{10}$, a cultura organizacional, o grau de variação tecnológica característica do setor, a velocidade de inovação e o tipo de conhecimento envolvido (tácito versus explícito) ${ }^{11,12}$. Alguns autores exploram as razões de externalização de atividades não centrais ao negócio, ou secundárias $^{13}$, outros ${ }^{14-17}$ apontam as estratégias de flexibilidade e volume como resposta a flutuações da demanda e à complexidade e variedade de tratamentos como determinantes para a externalização de atividades principais, centrais, que em organizações de saúde, significam "contacto directo com o usuário"18,19.

Na perspectiva institucional, as decisões make or buy revelam, muitas vezes, imitação das decisões do líder do setor ${ }^{20}$ devido a uma "inércia organizacional" na imitação do líder sem preocupações de eficiência ${ }^{21}$. Este tipo de isomorfismo pode servir propósitos de legitimação ${ }^{22}$, mas pode tornar-se perigoso à sobrevivência das organizações ${ }^{23}$. 


\section{Riscos e vantagens}

O compromisso com a fiabilidade ${ }^{24}$ das unidades de saúde confere a cada decisão um componente de risco que obriga a um processo de avaliação do mesmo ${ }^{25}$. Apesar de similares aos dos outros setores, os riscos mais frequentemente referidos na literatura sobre a terceirização na saúde são: a perda do controle do fornecedor e a perda de flexibilidade. No entanto, o benefício mais apontado é o da diminuição de custos, mesmo que apareça como consequência de outros benefícios, enumerados no Quadro 1.

\section{Monitorização da performance de atividades em terceirização}

Tal como referido, a literatura aponta para a importância da avaliação do risco no sucesso de uma estratégia de terceirização. Contudo, colocase a questão de como avaliar a performance/desempenho? Algumas recomendações ${ }^{4}$ podem funcionar como guidelines na tomada de decisão de externalização, mas não garantem o cumprimento de objetivos de performance. Existe a necessidade de investigação empírica sobre as medidas e od modelos de performance a adoptar perante as especificações correntes nos contratos de terceirização de modo a responder às questões em aberto. Por outro lado, a evolução da medição do desempenho coloca dúvidas, segundo vários autores $^{26-31}$, quanto à extensão da utilização das medidas interorganizacionais, da uniformização e da adequação das mesmas, não existindo consenso sobre o sistema a adoptar para medir o desempenho de atividades em terceirização. Uma via é a construção de indicadores específicos para cada tipo de atividade, numa perspectiva bottom-up, no terreno, avaliando os riscos concretos de cada atividade de modo a poder criar um sistema de medição e monitorização realmente eficaz ${ }^{32,33}$. A tentativa de avaliar essa possibilidade levou à realização deste estudo. A razão para monitorizar atividades externalizadas (e internas) é basicamente medir a conformidade com os níveis de serviço a um "custo aceitável" 34 . Alguns autores ${ }^{29}$ sugerem que a inexistência de estandardização de medidas de desempenho conjuntamente com a irregularidade de monitorização com demasiados indicadores leva ao aumento da importância das fortes relações de parceria. No entanto, as parcerias fortes demoram tempo a ser construídas e alimentadas e o insucesso da terceirização surge pela deficiente monitorização nesse compasso de espera ${ }^{35-}$ ${ }^{37}$. Qual deve ser, então, o ponto de partida para uma monitorização? Alguns autores ${ }^{34,38-42}$ alertam para a importância da abordagem de gestão de risco na investigação de gestão de operações mas deixam sem resposta algumas questões. Tal como referido antes, terceirização é assumir riscos. "Risco " é o resultado da combinação de três componentes: probabilidade de ocorrência de certo evento (positivo ou negativo), frequência e severidade das consequências $^{43}$. Logo, a gestão de risco é uma metodologia proactiva de análise dos riscos de atividades do passado, presente e, principalmente, do futuro de modo a lidar com a incerteza ${ }^{44}$. A postura de "esperar o inesperado" significa olhar para os processos, não pelos níveis de conformidade a atingir, mas pensando em possíveis falhas e nos impactos do serviço na organização ${ }^{45}$. Uma abordagem possível é seguir sete passos: contextualizar; identificar; analisar; quantificar; avaliar e ordenar por prioridades; tratar e gerir priorida-

Quadro 1. Riscos e Benefícios da Terceirização no setor da saúde

\begin{tabular}{|l|l|}
\hline \multicolumn{1}{|c|}{ Riscos } & \multicolumn{1}{c|}{ Benefícios } \\
\hline . Perda de controlo e flexibilidade & . Foco nas competências centrais e distintivas \\
\hline . Dependência do fornecedor & . Acesso a especialistas \\
\hline . Problemas de comunicação & . Redução de custos \\
\hline . Perda de reputação & . Adaptabilidade a necessidades internas \\
\hline . Exposição a violação de conformidade & . Capacidade de inovação \\
\hline . Custos "escondidos" & . Menor volatilidade no investimento tecnológico \\
\hline . Problemas de rotação e qualidade & . Resposta a aumento de volume e capacidade \\
\hline . Descontinuidade/inconsistência do serviço & . Melhor serviço ao utente \\
\hline . Diferenças culturais & . Benefícios públicos (acessibilidade) \\
\hline
\end{tabular}

Fonte: Elaboração própria com base em: Atun ${ }^{15}$; Augurzky e Scheuer ${ }^{46}$; Cesarotti e Di Silvio ${ }^{47}$; Chasin et al..$^{48}$; Dancer $^{49}$; Earl $^{50}$; England ${ }^{51}$; Giarraputo ${ }^{52}$; Goggin ${ }^{53}$; Greeno $^{54}$; Hazelwood et al. ${ }^{8}$; Liu et al. ${ }^{36} ;$ MacCutcheon e Griffin $^{55} ;$ Peisch $^{56}$; Renner e Palmer ${ }^{57}$; Roberts ${ }^{4}$; Schneller e Smeltzer ${ }^{58}$. 
des e, por fim, monitorizar e rever os riscos ${ }^{39}$. Outros autores ${ }^{40,59}$ enunciam um processo similar denominado "análise modal de falhas e efeitos” FMEA (Failure Mode Effects Analysis). A abordagem de gestão de risco parece ser a mais adequada em processos de terceirização em unidades de saúde onde a questão da fiabilidade leva à preocupação com a perda de controle do serviço e da qualidade. Este aspecto torna-se mais relevante quanto maior for o tempo de permanência do usuário, nomeadamente nos cuidados de longa duração como os Cuidados Continuados (CC) ${ }^{55}$.

\section{Metodologia}

A revisão prévia da literatura existente sobre terceirização em unidades de saúde deixa sem resposta algumas questões: QI.1 - Que razões levam uma unidade de saúde em início de atividade a adoptar uma estratégia de terceirização? QI.2 Como avaliar e seleccionar os fornecedores? QI.3 Como monitorizar a performance das atividades externalizadas? A escolha pelo método do estudo de caso resultou do próprio problema em investigação - a monitorização de performance de atividades em terceirização em unidades de saúde - e das QI anteriormente apresentadas. Este método é indicado para questões de "como" e "porquê", sendo uma inquirição empírica que investiga um fenômeno contemporâneo dentro de um contexto da vida real, em situações onde os comporta- mentos relevantes não podem ser manipulados e os limites entre o fenômeno e o contexto não se encontram claramente definidos, recorrendo a diferentes técnicas de recolha de dados, em simultâneo, e provenientes de diferentes tipos de fontes de evidência ${ }^{60}$. Permitindo um conhecimento aprofundado de um fenómeno ${ }^{61}$ é um método frequentemente usado em investigação na área da gestão, nomeadamente na operacional ${ }^{62}$. Mais idiossincrático do que generalizador, foi escolhido pelo seu carácter descritivo e exploratório, não para produzir declarações de causalidade, mas para apresentação de uma sequência lógica de conexão entre dados empíricos, problema/questões de investigação e resultados/conclusões.

De acordo com a revisão da literatura efectuada formulam-se dez proposições teóricas (PT) (Quadro 2). A escolha da unidade de análise resultou das proposições apresentadas agrupadas em cinco dimensões de análise: posicionamento estratégico da organização, ótica de criação de valor, motivos de decisão de terceirização, fases do processo de terceirização e sistema de monitorização das atividades externalizadas. Esta escolha obedeceu aos seguintes critérios de inclusão: ser uma unidade de saúde privada (por ser diferente o processo de tomada de decisão comparativamente às públicas), encontrar-se em início de atividade, prestando serviços marcadamente customizados (em que a customização se opõe à padronização pela adaptação total da prestação às necessidades e às preferências do usuário e

Quadro 2. Proposições Teóricas

\begin{tabular}{|l|}
\hline \multicolumn{1}{|c|}{ Proposições teóricas } \\
\hline $\begin{array}{l}\text { PT.1 - No início do ciclo de vida de uma organização, a decisão de externalizar atividades pode ser tomada por } \\
\text { motivos como restrições financeiras, velocidade de colocação no mercado, flexibilidade perante variações de } \\
\text { volume, obtenção de economias de escala, utilização da capacidade e falta de conhecimento interna }\end{array}$ \\
\hline PT.2 - As organizações são levadas a decisões de externalização por isomorfismo \\
\hline PT.3 - As atividades seleccionadas derivam do grau de especificidade, complexidade, âmbito e criticidade \\
\hline PT.4- A seleção depende do grau de proximidade da atividade com o utente/usuário \\
\hline PT.5 - A seleção deriva da avaliação do risco/benefício da terceirização de cada atividade \\
\hline $\begin{array}{l}\text { PT.6 - As relações que mais se afastam das parcerias envolvem menor confiança e respeitam a atividades } \\
\text { menos críticas }\end{array}$ \\
\hline $\begin{array}{l}\text { PT.7 - O insucesso de um processo de terceirização reside não só na má avaliação dos riscos e benefícios } \\
\text { associados a cada atividade, mas também na má gestão do risco durante todas as etapas do processo }\end{array}$ \\
\hline PT.8 - A terceirização falha por deficientemente gerida e monitorizada \\
\hline $\begin{array}{l}\text { PT.9 - As falhas de performance das atividades externalizadas comprometem o arranque de atividade por } \\
\text { maior exposição ao risco }\end{array}$ \\
\hline PT.10 - A monitorização deve estar inserida num programa de gestão de risco \\
\hline
\end{tabular}


caráter inovador $\left.{ }^{63}\right)$, e apresentar atividades em terceirização (clínicas e não clínicas). Assim, a unidade de saúde escolhida foi uma de CC fortemente diferenciada dos seus concorrentes pelo caráter inovador das soluções geriátricas individualizadas e pelos níveis de customização na prestação do serviço, para além de ser uma organização em início de atividade. Na recolha e no tratamento de dados foi seguido um protocolo de estudo e formada uma cadeia de evidência viabilizada pela triangulação das várias fontes de da$\operatorname{dos}^{60}$. De Abril a Outubro de 2008 foram efetuadas entrevistas semiestruturadas (ao administrador, ao diretor geral e ao de operações, ao diretor de marketing, ao consultor externo e a três gestores de departamento), analisada a documentação (regulamento interno, pedidos de propostas, propostas e cadernos de encargos, contratos, apresentações formais, comunicações internas, manuais dos fornecedores, fichas técnicas, regulamentação setorial, códigos de boas práticas, material promocional e press releases). Foi feita ainda observação direta não participante (procedimentos das atividades em terceirização $)^{64}$. Após codificação dos dados relevantes, procedeu-se à redução de $\operatorname{dados}^{65}$ de modo a poderem ser identificados padrões. Os resultados comparados com o suporte teórico de referência puderam, então, ser apresentados.

\section{Estudo de Caso}

\section{Posicionamento estratégico}

A CLB (denominação resultante de compromisso de confidencialidade) foi constituída no final de 2003 para actuação na área das residências assistidas e cuidados para pessoas idosas posicionando-se no mercado alvo de classe média e média-alta com uma unidade composta por: um serviço de avaliação geriátrica, um serviço de terapias, quatro pisos residenciais (136 camas) e duas unidades de dia com 30 lugares. Os entrevistados referiram como objetivos de mercado da CLB atingir no período de 7 a 9 anos uma quota de mercado de $15 \%$ a $20 \%$ e, num horizonte mais alargado, atingir 10 unidades em 20 anos. A CLB efectuou a análise da concorrência seguindo os parâmetros: tipologia da instituição, infraestruturas, caracterização dos usuários, estrutura dos recursos humanos, horário das visitas, preçário, motivação dos residentes, atendimento ao usuário, pontos fortes e fracos. Em todos os parâmetros, a CLB assumiu-se como um modelo inovador de prestação de cuidados de geriatria em Portugal. A CLB oferece, desde 24 de Abril de 2008, soluções abrangentes e especializadas nas áreas da avaliação geriátrica, dos cuidados de longoprazo e do alojamento, direcionadas para qualquer adulto que "valorize ou necessite de apoio para o desenvolvimento de atividades de subsistência no âmbito da vida do quotidiano”.

\section{Ótica de criação de valor}

Segundo os entrevistados as expectativas principais do usuário (e família) são: higiene das instalações e dos colaboradores; conforto e bom ambiente; segurança; e soluções de saúde. Assim, a CLB define-se como uma "unidade de saúde em ambiente hoteleiro" com especificidades que vão desde a restrita circulação de pessoas aos planos alimentares individuais e específicos, à supervisão permanente, de natureza técnica e clínica, e à grande proximidade entre técnico e usuário. Estas especificidades derivam do contexto da intervenção clínica de reabilitação, manutenção e prevenção que caracteriza a unidade em termos de cuidados de saúde e leva a adaptações do serviço hoteleiro. Perante o "rendilhado de necessidades", os "mesmos ingredientes originam diferentes receitas", oferecendo a CLB, um conjunto variado de serviços nas áreas da funcionalidade, saúde, higiene pessoal e alojamento, de modo a que as pessoas se agrupem nos diferentes pisos/núcleos por diferentes níveis de dependência. Os Módulos de Cuidados incluem: Avaliação Geriátrica Integral (AGI), cuidados de apoio pessoal, terapias integradas, apoio de âmbito social, cuidados de enfermagem, apoio médico de medicina geral, apoio de nutrição, medicação e cuidados de podologia. A AGI, feita no ingresso, estabelece um retrato abrangente das capacidades e limitações funcionais do usuário, podendo ser identificado o nível de dependência respectivo e estabelecidos objetivos e estratégias (periodicidade e intensidade) de intervenção, a constar no plano de cuidados sujeito a revisão nos períodos de reavaliação pré-estabelecidos ou sempre que ocorra alguma alteração do estado do usuário. O grau elevado de customização se reflete em: planos alimentares individuais definidos por nutricionista, dietas específicas individualizadas, planos de terapias individuais e possibilidade de atender a pedidos especiais do usuário, desde que salvaguardada a sua saúde e bemestar. A ótica de criação de valor é resumida por um entrevistado: $O$ que nos distingue dos outros operadores é estar ao nível máximo da customização de cuidados geriátricos.

\section{Motivos da decisão de terceirização}

O processo de decisão de terceirização foi analisado em três vertentes: a dos motivos e con- 
dicionantes, a da seleção das atividades propriamente ditas e a da avaliação dos riscos associados à externalização de cada uma das atividades. Foi recolhida evidência sobre motivações e sobre condicionantes (propensão ao risco, posição relativamente à concorrência, grau de inovação do negócio/setor, arquitetura do serviço/modularidade, cultura organizacional e o grau de formalização do conhecimento). Os motivos da decisão encontrados no caso CLB são os corroborados pela evidência sistematizada no Quadro 3. A focalização nas atividades que a organização "sabe fazer bem" a par da procura de especialistas que rapidamente dessem resposta de forma a não comprometerem o início da unidade foram, alegadamente, as razões primordiais que levaram a optar pela terceirização de atividades. Os critérios de seleção das atividades foram: o grau de especificidade, o grau de complexidade, o âmbito, a criticidade da atividade e a proximidade da atividade ao usuário externo. O principal critério foi a proximidade com o usuário seguindo a regra de inclusão: "Tudo o que não for entregue diretamente ao usuário é passível de ser externalizado". Foram externalizadas as atividades: menos específicas - no sentido em que pouca diferenciação da oferta não comprometeria a perso- nalização dos serviços internos; menos complexas - no sentido em que a as especificações e requisitos do serviço pudessem ser ditados pela CLB; de âmbito alargado - no sentido em que a periodicidade da transacção deveria ter caráter de permanência; e de média criticidade - no sentido em que se externalizaram as atividades secundárias da CLB, e pelo facto menos críticas, tendo, no entanto, alguma criticidade pela grande visibilidade perante o usuário externo. Assim, as atividades em terceirização destacam-se em sombreado na cadeia de valor da CLB representada na Figura 1.

\section{Avaliação dos riscos e benefícios \\ associados à externalização}

A avaliação dos riscos e benefícios resultantes da externalização das diferentes atividades foi decorrente do desenvolvimento da relação com o prestador. Houve, no entanto, a priori uma enumeração dos principais riscos, nomeadamente dos que se prendem com a saúde e o bemestar dos usuários que foi um dos pontos de partida para a elaboração dos cadernos de encargos (CE). Todos os entrevistados enumeraram vários riscos tais como: diferenças de cultura e valores; perda de controle do fornecedor; falhas de qualidade; insatisfação e reclamações;

Quadro 3. Motivos da decisão de externalizar na CLB

\begin{tabular}{|c|c|}
\hline Motivações & Evidência \\
\hline . Financeiras & . Restrições de custos (alegadamente secundário) \\
\hline . Tecnológicas & $\begin{array}{l}\text { Procura de expertise, especialistas, complexidade da gestão logística de } \\
\text { algumas atividades }\end{array}$ \\
\hline . Estratégicas & $\begin{array}{l}\text { Flexibilidade, escassez de tempo, velocidade de colocação no mercado, } \\
\text { focalização nas atividades core, aparecem como factores principais }\end{array}$ \\
\hline . Políticas/Institucionais & . As parcerias não influenciaram a decisão \\
\hline Condicionantes & Evidência \\
\hline . Propensão ao risco & . Necessidade de mitigar riscos pela terceirização, embora não especificados \\
\hline . Posição relativamente à concorrência & . Os modelos adoptados pela concorrência não influenciaram a decisão \\
\hline . Grau de inovação do negócio/setor & $\begin{array}{l}\text { Relativamente à concorrência, a CLB assume-se como inovadora no modelo } \\
\text { de cuidados geriátricos }\end{array}$ \\
\hline - Arquitectura do serviço/ Modularidade & $\begin{array}{l}\text { A oferta de serviços estrutura-se por módulos e o espaço encontra-se } \\
\text { adequado à mesma }\end{array}$ \\
\hline . Cultura organizacional & $\begin{array}{l}\text {. A figura que o utente vê deve ser identificada, em regra, como "residente" } \\
\text { CLB }\end{array}$ \\
\hline - Grau formalização conhecimento & $\begin{array}{l}\text {. A informalidade existente não obsta o registo e reporting. Existe evidência na } \\
\text { formalização crescente ao nível da elaboração de manuais de procedimentos, } \\
\text { ainda não implementados em todos os serviços, embora a comunicação } \\
\text { interna seja mais verbal do que escrita; Cultura de reporting incipiente. }\end{array}$ \\
\hline
\end{tabular}

Fonte: Elaboração própria 
Figura 1. Cadeia de valor da CLB

não cumprimento do contrato/CE; riscos de perda de competências críticas (saúde e segurança dos usuários e risco de dependência do fornecedor). No que respeita aos benefícios, foram de mais rápida enumeração os seguintes: acesso a know-how específico; eliminação de stocks; libertação de recursos; aprendizagem com o fornecedor; partilha de responsabilidades; concentração de competências no que é central e crítico ao negócio e obtenção de flexibilidade.

\section{Fases do processo de terceirização}

O processo de seleção de fornecedores resultou de uma busca nacional de prestadores seguindo os critérios de dimensão/estrutura, idade (estarem no mercado há "algum tempo") e oferta de serviços. Foram analisados em pormenor os CE respeitantes aos serviços de limpeza e alimentação por serem mais estruturados que os demais. Da análise efectuada ressalta o facto de ambos os CE incidirem mais em especificações de input e de processo tais como: especificações técnicas dos materiais a utilizar; especificações de preço e quantidade dos recursos (humanos, equipementos e materiais) e respectiva variação em função das diferentes taxas de ocupação; especificações ténues sobre o nível de competências técnicas do pessoal e especificações de serviço. No que respeita a especificações de output, existe menção, nos CE, apenas quanto a alguns resultados de limpeza esperados de caráter ambíguo e não traduzidos em objetivos mensuráveis. Não existe, contudo, menção nos CE a sistemas de monitorização a adoptar em cada serviço, à ex- cepção do disposto por lei, no caso da alimentação, no que se refere à obrigatoriedade de cumprimento das normas HACCP (Hazard Analisys of Crítical Control Points). Reunidos os candidatos foram enviados pedidos de propostas solicitando detalhe sobre qualidade e preços das matérias-primas, quantidade, custo e categorias profissionais da mão-de-obra, custos administrativos afectos ao projecto e certificações de qualidade. Na seleção do fornecedor foram também tidos em conta factores subjetivos como a "empatia" e a forma de estar do fornecedor. Quanto ao tipo de relação contratual, este variou conforme a duração, o tipo de cláusulas, o nível de confiança pretendido e o comprometimento com resultados. Todos os contratos foram elaborados para a duração de um ano. Os contratos analisados podem ser considerados clássicos, com uma estrutura-tipo. Quanto ao nível de confiança, em termos contratuais, a CLB não incluiu especificações sobre as medidas que podem ser tomadas pelo contratador no caso de falha de fornecimento. Este aspecto não pode ser dissociado do comprometimento com resultados, remetendo para a necessidade de vinculação ao disposto no CE. Se na alimentação o detalhe foi negociado e ajustado, tal não ocorreu com o serviço de limpeza. Foi seleccionado o candidato que apresentava o menor preço.

\section{Análise do risco e monitorização das atividades externalizadas}

Foram identificados os atores de monitorização para cada atividade em terceirização em 
vários momentos de tempo de modo a tentar criar um historial para observação de processos. Relativamente às possibilidades/vias de monitorização, os serviços em terceirização foram analisados de acordo com: especificações do contrato; monitorização da performance pelo contratador; eficiência do contratado; avaliação do serviço pelo usuário final; alinhamento da entrega e dos resultados e responsabilização pelos resultados. Estes, demonstram uma inexistência de monitorização efectiva das atividades de limpeza e de refeições bem como uma necessidade crescente de implementação de um plano de monitorização para cada atividade. A evidência recolhida, posta em confronto com a documentação analisada, indica uma série de falhas. Foram encontradas desfasagens entre o acordado e o realizado nas áreas de alimentação e limpeza, denotando maiores índices de insatisfação, sendo as que o usuário final mais valoriza. Estas desfasagens incitaram a um processo de ajuste de especificações de input e de estudo dos processos para a optimização dos recursos e a melhoria dos tempos e da qualidade do serviço, resultando num novo dimensionamento da equipe, numa adequação maior das pessoas às tarefas, num novo layout, numa agenda melhor definida, num apuro de custos diferente e numa clara necessidade de monitorização. Foram analisadas as falhas segundo o instrumento de gestão de risco FMEA, de modo a criar um ponto de partida para a elaboração de um plano de monitorização.

\section{FMEA para o serviço de limpeza}

As falhas enumeradas pelos entrevistados foram completadas pela observação direta e confrontadas com os requisitos constantes do CE. Quatro tipos de falhas (qualidade, tempo, custo e flexibilidade) foram dispostas em diagramas de causa/efeito numa tentativa de explicar a má performance da atividade externalizada. O diagnóstico de falhas seguiu a lógica da análise do nível de risco associado a cada procedimento de limpeza (PL), uma vez que na unidade CLB os quatro níveis de risco de contaminação se encontram bem definidos, estando a cada espaço atribuído um nível de risco. Assim, em cada PL procurou-se identificar os modos potenciais de falha, os respectivos efeitos, as causas e os mecanismos atuais de controle. Garantiu-se, para cada serviço, que as grades de ponderação utilizadas fossem produto de sessões de trabalho com uma equipe multidisciplinar interna à CLB, de modo que foi a própria organização que determinou os diferentes níveis de aceitação nos diferentes fatores: probabilidade de detecção, probabilida- de de ocorrência e grau de severidade de ocorrência. Na atribuição da ponderação a cada falha teve-se em conta valores e resultados realistas de referência. As falhas encontradas encontram-se sistematizadas numa tabela FMEA (de que é exemplo a Tabela 1). Os RPN (Risk Priority Numbers) encontrados (por multiplicação dos valores de Deteção, Severidade e Ocorrência) revelam uma necessidade de atuação que não passará apenas pela implementação de um sistema de monitorização. A CLB tem vindo a tentar colmatar a falta de formação do pessoal externo (prevista, mas nunca cumprida pelo contratado) com ações de formação interna, nomeadamente sobre regras de controle de infecções que vão desde os procedimentos básicos de higiene pessoal à forma de limpar e desinfectar.

Do exposto resulta a necessidade de criação de um plano de monitorização, com ator(es) e periodicidade bem definidos tal como o lado direito da tabela propõe. Os resultados previstos após implementação do sistema de monitorização revelam uma intenção de melhoria contínua, pelo que a necessidade de realização de novo FMEA não se colocará apenas aos casos em que o RPN ultrapassou o valor 70 de referência.

\section{FMEA para o serviço de refeições}

A satisfação e o agrado do usuário iniciais foram-se alterando, segundo os entrevistados, em função das mudanças ocorridas na equipe prestadora. O serviço, considerado de maior satisfação passou a ser um dos mais problemáticos e com reclamações diárias. Acresce o facto de ser um serviço cuja má prestação é atribuída, pelo usuário, ao contratador. O tipo de relação encetado com este prestador, que no início se aproximou da parceria, pelo trabalho conjunto de definição da estrutura de meios adequada sofreu alterações cadenciadas pela frequência da mudança da equipe, o que debilitou a relação estabelecida. A crescente complexidade resultante quer da implementação de planos alimentares individuais específicos, quer dos múltiplos acabamentos de pratos individuais demonstraram uma desfasagem entre o disposto no CE e a realidade, fazendo repensar a quantidade e a qualidade da composição da equipe e até o layout da cozinha.

O percurso de análise de falhas seguiu a cadeia de subprocessos do serviço. De acordo com a observação efetuada, os resultados da redução de dados das entrevistas e da documentação analisada (CE, Codex Alimentarius, Código de boas práticas da restauração coletiva e proposta do contratado) as falhas encontradas foram pontuadas de acordo com a grade de ponderação. 
Tabela 1. FMEA para o serviço de limpeza

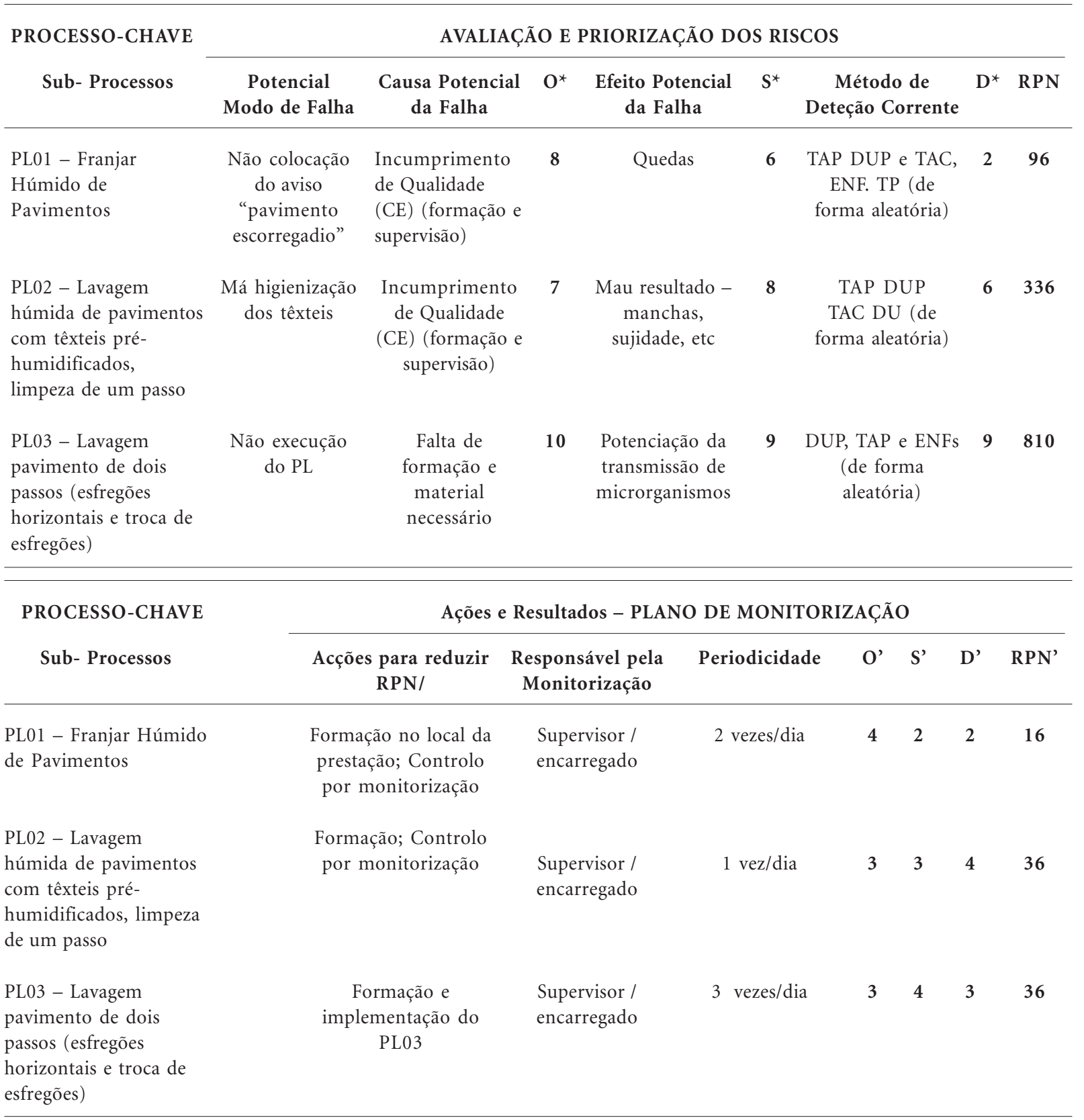

Assim, o modo de falha encontrado com maior grau de risco associado foi o não cumprimento das fichas técnicas, onde pesa não a frequência em que ocorre, mas, principalmente, a severidade inerente traduzida na insatisfação do usuário e nos riscos para a sua saúde, o que se agrava em tratando-se de doentes com doenças crónicas cuja variação se encontre muito associada a variações da alimentação. Todas as restantes falhas se encontram aliadas à necessidade de implementação de um plano de monitorização, bem como soluções de formação e melhoria da comunicação, de modo que a informação flua em tempo útil, possibilitando ganhos em planeamento e qualidade do serviço.

\section{Conclusões}

No processo de tomada de decisão de externalização na CLB, o fator tempo e velocidade de entrada (speed to market) foram determinantes uma 
vez que a focalização no essencial do negócio levou a evitar "distrações" sobre o acessório. Tornou-se, assim, urgente encontrar especialistas como forma de colmatar a falta de conhecimento interno, para todas as atividades sem contacto direto com o usuário (o que valida a PT.4) libertando os recursos para a focalização na atividade principal/nuclear "cuidar do usuário". As razões financeiras, alegadamente secundárias, prevaleceram na escolha entre propostas similares e não negociadas em detalhe. As restantes razões encontradas enfatizam a obtenção de flexibilidade para a qual contribui a própria arquitetura do serviço e a concepção do espaço. Deste modo, num início de actividade, a terceirização surge por motivos como restrições financeiras, velocidade de colocação no mercado, flexibilidade perante variações de volume, obtenção de economias de escala, utilização da capacidade e falta de conhecimento interno, o que valida a proposição teórica PT.1. Inversamente, a evidência do caso não foi suficiente para validar a $P T .2$, não tendo sido encontrados indícios de isomorfismo ou posição de "seguidor" do líder.

O processo de seleção de atividades, embora sofrendo acertos de reavaliação, seguiu os critérios de especificidade, complexidade, âmbito e criticidade das atividades, o que valida a proposição teórica $P T .3$ formulada. A avaliação efetuada de riscos e benefícios, por não ter sido prévia e sistematizada não condicionou a seleção de atividades de forma peremtória, pelo que a evidência recolhida não é suficiente para validar a proposição teórica $P T .5$. O facto é que a CLB considerou algumas atividades (refeições, limpeza e farmácia) como tendo praticamente o mesmo nível de criticidade, pelo que a proposição teórica PT.6 não pôde, no caso CLB, ser validada. As atividades cuja insatisfação com a opção de terceirização é crescente poderão ver o prestador substituído ou voltar a ser internalizadas. Em todo o processo não houve uma avaliação sistemática de riscos e benefícios pelo que o estudo do caso CLB valida a proposição teórica PT.7. Por outro lado, não foi possível validar a proposição teórica PT.8 que carece de ver implementado um sistema de monitorização completo para que se possa avaliar o sucesso da decisão de externalização para cada atividade. Não tendo sido definidos e acordados níveis de serviço para cada atividade, os reajustes refletiram-se em custos acrescidos quer em consultoria externa, formação interna, tempo despendido em reorganização de processos, contratação, formação e renegociação de alguns parâmetros de serviço. Os diferentes níveis de detalhe no modelo que serviu de base aos pedidos de proposta levaram a que a negociação ficasse empobrecida, resultando em baixo comprometimento do prestador e inexistente responsabilização pelos resultados.

A proposição teórica PT.9 não pôde, neste caso, ser validada uma vez que o início, propriamente dito, não ficou comprometido. Mas, não tendo sido assegurado o controle de falhas, os efeitos em termos de fidelização, reorganização interna de processos e até mesmo de avaliação de substituição de prestadores e reequacionamento da questão make or buy, não foram os esperados em grande parte das atividades. O processo de $F M E A$, ao ser proactivo e cíclico, leva a que a monitorização possa ser um dos outputs de um programa de gestão de risco, o que valida a proposição teórica PT.10.

Em suma, perante um início ambicioso de um serviço inovador, num mercado assomado por entradas simultâneas de vários concorrentes, a terceirização tem um efeito propulsor ao permitir a concentração de esforços internos no desenvolvimento de competências críticas ao negócio, alavancar recursos e imprimir a necessária flexibilidade a ajustes no modelo de negócio e na velocidade de penetração no mercado.

No entanto, esta velocidade pode conferir um ritmo nefasto em termos não só de tomada de decisão, mas, sobretudo, por resultar numa forma demasiadamente superficial de encarar todo o processo desde o sourcing, benchmarking externo, pedidos de propostas, negociação, contratação e monitorização. O recurso a uma abordagem de gestão de risco permite, não só diminuir essa superficialidade na análise, como construir a base de um sistema capaz de acompanhar a performance do fornecedor de forma a construir indicadores realistas, possibilitando a responsabilização pelas ações de correção e, até, reequacionar a opção de terceirização para cada atividade segundo parâmetros de tempo, qualidade, custo e flexibilidade.

Não tivemos, neste estudo, o intuito de produzir declarações de causalidade, mas de sistematização da informação tácita que evidencia quatro ordens de falhas: de custo, de qualidade, de tempo e de flexibilidade. As conclusões retiradas do estudo do caso CLB pretendem, ao serem idiossincráticas, contribuir para aumentar a compreensão da ocorrência do fenômeno dentro das circunstâncias que serviram como critérios de inclusão, mas carecem de carácter generalizador, pelo que só a replicação do estudo a outros casos com os mesmos critérios de inclusão poderá permitir. O estudo deste caso sugere, ainda, algumas perspetivas de investigação futura tal como o estudo 
de implementação de um sistema de medição e monitorização da performance de todas as atividades em terceirização e estudo dos respetivos efeitos na performance global da organização.

\section{Colaboradores}

CM Guimarães foi responsável pela metodologia, revisão bibliográfica, tratamento e análise dos dados coletados e redação do artigo. JC Carvalho orientou a elaboração, contribuiu com conhecimentos na área de terceirização e de Supply Chain Management e revisou o artigo.

\section{Referências}

1. Brown D, Wilson S. The Black Book of Outsourcing: How to Manage The Changes, Challenges and Opportunities. Wiley: John \& Sons Incorporated; 2005.

2. Quinn JB, Hilmer FG. Strategic Outsourcing. Sloan Management Review 1994; 35(4):43-55.

3. Quinn JB. Outsourcing Innovation: The New Engine of Growth. Sloan Management Review 2000; 41(4):13-29.

4. Roberts V. Managing Strategic Outsourcing in the Healthcare Industry. Journal of Healthcare Management 2001; 46(4):239-249.

5. Linder JC. Transformational Outsourcing. MIT Sloan Management Review 2004; 45(2):58.

6. Linder JC. Terceirização para a Mudança Radical: Uma Abordagem Sustentada para a Transformação da Empresa. Lisboa: Monitor Projectos e Edições Lda; 2008

7. Heavisides R. Output based Facilities Management Specifications in the National Health Service: Literature review and directional outcomes. Occupier.org Working Paper 1, Sheffield 2001. [acessado 2012 abr 1]. Disponível em: http://www.occupier.org/uploads/ articles/1working_paper1.pdf

8. Hazelwood SE, Hazelwood AC, Cook ED. Possibilities and Pitfalls of Outsourcing. Healthcare Financial Management 2005; 59(10):44-48.

9. Kremic TO, Tuckel I, Rom WO. Outsourcing decision support: a survey of benefits, risks, and decision factors. Supply Chain Management: An International Journal 2006; 11(6):467-482.

10. Simchi-Levi D, Kaminsky P, Simchi-Levi E. Designing \& Managing The Supply Chain, Concepts, Strategies \& Case Studies. $2^{\text {nd }}$ Ed. New York: McGrawHill; 2003.

11. Nonaka I. A Dynamic Theory of Organizational Knowledge Creation. Organization Science 1994; 5(1):14-37.

12. Perrons RK, Platts K. The role of clockspeed in outsourcing decisions for new technologies: insights from the prisioner's dilemma. Industrial Management \& Data Systems 2004; 104(7):624-632.

13. Conner KR, Prahalad CK. A Resource-Based Theory of the Firm: Knowledge versus Opportunism. Organization Science 1996; 7(5):477-501.
14. Wigglesworth K, Zelcer J. The healthcare supply chain: Applying best-practice remedies to the healthcare sector. In: Gattorna J, editor. Strategic Supply Chain Alignment - Best Practice in Supply Chain Management. London; Gower Publishing Limited; 1998.

15. Atun R. Privatization as descentralization strategy. Report to the World Health Organization Regional Office for Europe 2006; 14:246-271.

16. Campos AC. Decentralization and privatization in Portuguese health reform. Revista Portuguesa de Saúde Pública 2004; 4(7):7-20.

17. Jack EP, Powers TL. Managerial perceptions on volume flexible strategies and performance in health care services. Management Research News 2006; 29(5):228-241.

18. Young S. Terceirização: two case studies from the Victorian public hospital sector. Australian Health Review 2007; 31(1):140-149.

19. Young S. Outsourcing: uncovering the complexity of the decision. International Public Management Journal 2007; 10(3):307-325.

20. Meyer JW, Scott WR. Organizational environments: Ritual and rationality. Newbury Park: Sage Publications; 1992.

21. Hannan MT, Freeman JH. Structural inertia and organizational change. American Sociological Review 1984; 49(2):149-164.

22. Martin A, Bourgeois C. Le role des facteurs institutionnels dans la decision d'externaliser. Management International 2007; 11(4):17-29.

23. DiMaggio PJ, Powel WW. Institutional isomorphism and collective rationality. American Sociological Review 1983; 48(2):147-160.

24. Weick KE, Sutcliffe KM. Managing the unexpected: assuring high performance in an age of complexity. San Francisco: Jossey-Bass; 2001.

25. Arino AJ, dala Torre, Ring P. Relational quality: managing trust in corporate alliances. California Management Review 2001; 44(1):109-131.

26. De Toni AF, Fornasier A, Montagner M, Nonino F. A performance measurement system for facility management: The case study of a medical service authority. International Journal of Productivity and Performance Management 2007; 56(5/6):417-435. 
27. Duclos LK, Vokurka RJ, Lummus RR. A conceptual model of supply chain flexibility. Industrial Management \& Data Systems 2003; 103(5/6):446-456.

28. Folan P, Browne J. A review of performance measurement: Towards performance management. Computers in Industry 2005; 56(7):663-680.

29. Gunasekaran A, Patel C, Tirtiroglu E. Performance measures and metrics in a supply chain environment. International Journal of Operations \& Production Management 2001; 21(1/2):71-87.

30. Henri JF. Are your performance measurement systems truly performing? CMA Management 2006; 80(7):31-35.

31. Neely A. The search for meaningful measures. Management Services 2007; 51(2):14-17.

32. Loosemore M, Hsin YY. Customer-focused benchmarking for facilities management. Facilities 2001; 19(13/14):464-475.

33. Meyer MW. Rethinking Performance Measurement: Beyond the Balanced Scorecard. Cambridge: Cambridge University Press; 2002.

34. Rushton A, Walker S. International logistics and supply chain outsourcing: from local to global. London: Kogan Page Limited; 2007.

35. Abramson WB. Monitoring and evaluation of contracts for health service delivery in Costa Rica. Health Policy and Planning 2001; 6(4):404-411.

36. Liu X, Hotchkies DR, Bose S. The impact of contracting-out on health system performance: A conceptual framework. Health Policy 2007; 82(2):200-211.

37. Siddiqi S, Masud TI, Sabri B. Contracting but not without caution: experience with outsourcing of health services in countries of the Eastern Mediterranean Region. Bulletin of the World Health Organization 2006, 84(11):867-875.

38. McCormack P. The FSA approach to the supervision of outsourcing. Journal of Financial and Compliance 2003; 11(2):113-120.

39. Okoroh MI, Gombera PP, Ilozor BD. Managing FM (support services): business risks in the healthcare sector. Facilities 2002; 20(1/2):41-51.

40. Welborn C. Using FMEA to Assess Outsourcing Risk. Quality Progress 2007; 40(8):17-21.

41. Whitmore HB. You've Outsourced The Operation, but Have You Outsourced the Risk? Financial Executive 2006; 22(9):41-44.

42. Ritchie B, Brindley C. An emergent framework for supply chain risk management and performance measurement. Journal of the Operational Research Society 2007; 58:1398-1411.

43. Federation of European Risk Management Associations (FERMA). Norma de Gestão de Riscos. 2003. [site na Internet]. [acessado 2012 abr 20]. Disponível em: http://www.ferma.eu/wp-content/uploads/2011/11/arisk-management-standard-portuguese-version.pdf

44. Shohet I, Lavy S. Healthcare facilities management: state of the art review. Facilities 2004; 22(7/8):210220.

45. Atkinson W. Supply Chain Management: New Opportunities for Risk Managers. Risk Management 2006; 53(6):10-15.

46. Augurzky B, Scheuer M. Outsourcing in the German Hospital Sector. The Service Industries Journal 2007; 27(3):263-277.
47. Cezarotti V, Di Silvio B. Quality management standards for facility services in the Italian health care sector. Int J Health Care Qual Assur Inc Leadersh Health Serv 2006; 19(6):451-462.

48. Chasin BS, Elliot SP, Klotz SA. Medical Errors Arising from Outsourcing Laboratory and Radiology Services. Am J Med. 2007; 120(9):819.e9-11.

49. Dancer SJ. Mopping up hospital infection. J Hosp Infect 1999; 43(2):85-100.

50. Earl MJ. The risk of outsourcing IT. Sloan Management Review 1996; 37(3):26-32.

51. England RE. Experience of contracting with the private sector: a selective review, DFID Health Systems Resource Center 2004. [site da Internet]. [acessado 2012 abr 20]. Disponível em: http://www.nihfw.org/ $\mathrm{WBI} /$ docs/India\%20Flagship\%20sessions/Contracting/Exp $\% 20$ of $\% 20$ contracting $\% 20$ with $\% 20$ pvt $\%$ 20sector-DFID\%20paper.pdf

52. Giarraputo D. In-House Versus Off-Site Sterilization. Hosp Mater Manage Q 1990; 12(2):49-55.

53. Goggins R. Hazards of Cleaning. Professional Safety 2007; 52(3):20-27.

54. Greeno R. Hospitals take on the risk as they turn to hospitals programs. Managed Healthcare Executive 2001; 11(5):48-50.

55. MacCutcheon M, Griffin K. When Outsourcing Makes Cents. Post-Acute Care 2002; 5(4):32.

56. Peisch R. When outsourcing goes awry. Harvard Business Review. 1995; 73(3):24-30.

57. Renner C, Palmer E. Outsourcing to increase service capacity in a New Zealand Hospital. J Manag Med 1999; 13(5):325-338.

58. Schneller ES, Smeltzer LR. Strategic Management of the Health Care Supply Chain. Jossey-Bass: San Francisco; 2006.

59. Gunawardane G. Measuring reliability of service systems using failure rates: variations and extensions. The International Journal of Quality \& Reliability Management 2004; 21(4/5):580-590.

60. Yin RK. Case study research: design and methods. $3^{\text {rd }}$ ed. Thousand Oaks: Sage Publications Inc; 2003

61. Flyvbjerg B. Five Misunderstandings About Case Study Research. Qualitative Inquiry 2006; 12(2):219-245.

62. Voss C, Tsikriktsis N, Frohlich M. Case research in operations management. International Journal of Operations \& Production Management 2002; 22(2): 195-219.

63. Silvestro R. The manufacturing TQM and service quality literatures: synergistic or conflicting paradigms. International Journal of Quality \& Reliability Management 1998; 15(3):303-328.

64. Saunders M, Lewis P, Thornhill A. Research Methods for Business Students. 4rd ed. Prentice Hall, 2007.

65. Miles MB, Huberman AM. Qualitative Data Analysis. Thousand Oaks: Sage Publications. Inc; 1994.

Artigo apresentado em 13/04/2011

Aprovado em 17/03/2011

Versão final apresentada em 27/05/2011 